\title{
Bitterness and antibacterial activities of constituents from Evodia rutaecarpa
}

\author{
Xiaoguang Liang ${ }^{1 \dagger}$, Bo $\mathrm{Li}^{2+}$, Fei Wu', Tingzhao $\mathrm{Li}^{3}$, Youjie Wang ${ }^{1}$, Qiang Ma and Shuang Liang ${ }^{1 *}$
}

\begin{abstract}
Background: Bitter herbs are important in Traditional Chinese Medicine and the Electronic Tongue (e-Tongue) is an instrument that can be trained to evaluate bitterness of bitter herbs and their constituents. The aim of this research was to evaluate bitterness of limonoids and alkaloids from Evodia rutaecarpa to demonstrate that they are main bitter material basic of E. rutaecarpa.

Methods: Nine compounds, including limonoids, indoloquinazoline alkaloids and quinolone alkaloids, were isolated, identified and analyzed by the e-Tongue. Additionally, the antibacterial activities of the nine compounds were evaluated against $E$. coli and S. aureus.

Results: All the nine compounds had bitter taste and antibacterial activities to some extent. Among them, limonoids, which were the bitterest compounds, had greater antibacterial activities than alkaloids. And there is a positive correlation between bitter taste and antibacterial activities.

Conclusions: It was confirmed in our study that limonoids, indoloquinazoline alkaloids and quinolone alkaloids are main bitter material basic of E. rutaecarpa based on two evaluation methods of e-Tongue and antibacterial experiment. In addition, the e-Tongue technique is a suitable new method to measure bitter degree in herbs.
\end{abstract}

Keywords: Evodia rutaecarpa, Electronic Tongue, Bitterness, Antibacterial activity

\section{Background}

Bitter herbs are important in Traditional Chinese Medicine (TCM). According to the Chinese Pharmacopoeia 2010 edition, there are 177 species of bitter herbs, including many famous species, such as Coptis chinensis, Evodia rutaecarpa and Rheum officinale [1]. Based on the traditional theory and modern pharmacology, bitter herbs commonly possess widely pharmacological activities, including antibacterial, antitumor, regulating blood sugar level [2-5].

However, few studies have confirmed the correlation between the presence of bitter constituents in herbs and their pharmacological activities, mainly because there are no adequate methods to evaluate bitter constituents. Sensory panels are difficult to perform because of the possibility of health risks, and animal studies cannot be

\footnotetext{
* Correspondence: Is7312@163.com

${ }^{\dagger}$ Equal contributors

'Engineering Research Center of Modern Preparation Technology of TCM, Ministry of Education, Shanghai University of Traditional Chinese Medicine, 1200 Cailun Road, Shanghai 201203, People's Republic of China

Full list of author information is available at the end of the article
}

adequately controlled. The Electronic Tongue (e-Tongue) is an instrument that has been used to evaluate taste. In the past 20 years, the e-Tongue has been used to evaluate flavors in the food industry [6-8]. At the same time, pharmacy researchers began to use it in pharmaceutical research [9-12].

In a previous study, our laboratory evaluated the bitterness of berberine hydrochloride using the e-Tongue [13]. The study revealed that there were no differences between the e-Tongue data and the results obtained from a sensory taste panel, and e-Tongue is a suitable tool evaluating bitter constituents from TCM. Recently, we performed a study to evaluate E. rutaecarpa (Juss.) Benth., commonly referred to as "Wuzhuyu" in China. This herb, which has a spicy and bitter taste, has been used in the treatment of gastrointestinal disorders, abdominal pain, headache, and dysentery for thousands of years. Modern pharmacological studies have shown that the herb has antibacterial, anti-inflammatory, antiinfectious, and cytotoxic activities [14, 15]. Previous studies suggested that quinazolinedione alkaloids and 
limonoids are the most important effective components in E. rutaecarpa [16]. Among them, limonoids are representative natural bitter compounds.

In this paper, we describe the isolation, structural elucidation, bitterness evaluation using e-Tongue, and antibacterial activity testing of bitter constituents from E. rutaecarpa, and the correlation analysis between bitterness and antibacterial activities.

\section{Methods}

\section{Plant materials and chemicals}

Evodia rutaecarpa (Juss.) Benth. (No. 20130901) was purchased from Sichuan (China) and identified as Evodia rutaecarpa (Juss.) Benth. by Professor Zhi-li Zhao of the Shanghai University of Traditional Chinese Medicine (Shanghai, China). Materials for column chromatography included silica gel (10-40 $\mu \mathrm{m}$; Huiyou Silical Gel Development Co. Ltd. Yantai, P. R. China), Sephadex LH-20 (40-70 $\mu \mathrm{m}$; Amersham Pharmacia Biotech AB, Uppsala, Sweden), and reversed-phase $C_{18}$ silica gel (50 $\mu \mathrm{m}$; YMC, MA, U.S.A.). Acetonitrile (HPLC grade) was purchased from Merck KGaA (Darmstadt, Germany). Ultrahigh purified water used in this study was prepared in a Milli-Q water purification system (Millipore Corp., Billerica, MA, USA). All other reagents and chemicals, which were of analytical grade, were purchased from Sinopharm Chemical Reagent Company Ltd. (Shanghai, China).

\section{Bacterial strains and cultivation}

Escherichia coli (CMCCB44113) and Staphylococcus aureus (CMCCB26003) were obtained from the Chinese National Center for Medical Culture Collections (CMCC, Beijing, China). Stock cultures in $25 \%$ glycerol were maintained at $-80{ }^{\circ} \mathrm{C}$. Bacterial strains were inoculated into lysogeny broth (LB) and grown for $6 \mathrm{~h}$ at $37^{\circ} \mathrm{C}$ under constant shaking (165 rpm). Subsequently, the bacterial cultures were centrifuged at $3000 \mathrm{rpm}$ for $5 \mathrm{~min}$ and washed three times with $10 \mathrm{mM}$ phosphatebuffered solution (PBS, $\mathrm{pH}$ 7.4). The resulting pellets were re-suspended in PBS and inoculated into agar plates. Bacterial densities were determined using a scattered light turbidimeter; bacterial amount was derived from bacterial density.

\section{Equipments}

An $\alpha$-Astree liquid and taste analyzer (e-Tongue, Alpha Mos Inc) was coupled to seven taste sensors, an LS16 auto-sampler, and a reference electrode $(\mathrm{Ag} / \mathrm{AgCl}$, Alpha Mos Inc.). The system was equipped with a data acquisition and analysis software. The taste sensors included ZZ14601, AB11303, GA13207, BB14005, C13602, DA10905, and JE13101. NMR spectra were recorded in an Avance 500 NMR spectrometer with TMS as the standard. ESI-MS were measured in an Agilent LC/MSD Trap XCT mass spectrometer; HR-ESI-MS were measured in a Q-TOF micro mass spectrometer (Waters, USA). Optical rotations were acquired with a PerkinElmer 341 polarimeter, whereas IR spectra were recorded in a Bruker Vector 22 spectrometer with $\mathrm{KBr}$ pellets. Optical density was determined in an ELISA reader (CLX800-BioTek Instruments, USA).

\section{Extraction and Isolation of compounds 1-9}

Dried and powdered E. rutaecarpa fruits $(6.0 \mathrm{~kg})$ were extracted twice with methanol $(48 \mathrm{~L})$ under reflux for $2 \mathrm{~h}$ each time. Methanol was evaporated under vacuum, and the extract was suspended in water and successively partitioned with petroleum ether $(3 \times 10 \mathrm{~L}), \mathrm{CH}_{2} \mathrm{Cl}_{2}$ $(3 \times 10 \mathrm{~L})$, EtOAc $(3 \times 10 \mathrm{~L})$, and n-butanol $(3 \times 10 \mathrm{~L})$. The $\mathrm{CH}_{2} \mathrm{Cl}_{2}$ extract was subjected to $\mathrm{CC}$ on silica gel and eluted with gradient $\mathrm{CH}_{2} \mathrm{Cl}_{2}-\mathrm{MeOH}$. The eluates were further subjected to on silica gel to give Compounds $\mathbf{1}, \mathbf{2}, \mathbf{3}, \mathbf{4}, \mathbf{5}$ and $\mathbf{8}$. The EtOAc extract was also subjected to $\mathrm{CC}$ on silica gel and eluted successively with gradient $\mathrm{CH}_{2} \mathrm{Cl}_{2}-\mathrm{MeOH}$ mixtures of increasing polarity. The eluates were rechromatographed on ODS $\left(\mathrm{CH}_{3} \mathrm{OH}-\mathrm{H}_{2} \mathrm{O}\right)$ followed by Sephadex LH-20 with $\mathrm{CH}_{2} \mathrm{Cl}_{2}-\mathrm{MeOH}$ (1:1) to give Compounds 6, 7 and 9.

\section{Chromatographic and mass spectrometric conditions} Chromatography was performed on an HPLC system (Thermo Finnigan, Thermo Corp., USA) equipped with a conditioned auto-sampler maintained at $10{ }^{\circ} \mathrm{C}$ and an Agilent ZORBAX Eclipse $\mathrm{XDB}^{-\mathrm{C}_{18}}$ column $(4.6 \times 150 \mathrm{~mm}, 5 \mu \mathrm{m}$, Agilent Corp., USA) maintained at $40{ }^{\circ} \mathrm{C}$. A gradient elution was performed using acetonitrile (reagent $\mathrm{A}$ ) and $0.1 \%$ acetic acid solution (reagent B) as the mobile phase: $10 \%$ reagent $\mathrm{A}$ from 0 to $0.5 \mathrm{~min} ; 10$ to $50 \%$ reagent A from 0.5 to $12 \mathrm{~min}$, 50 to $95 \%$ reagent $\mathrm{A}$ from 12 to $17 \mathrm{~min}$, and $95 \%$ reagent A for $1 \mathrm{~min}$. Reagent A was reduced to $10 \%$ for column equilibration. The total cycle time was $23 \mathrm{~min}$ with a flow rate of $0.3 \mathrm{ml} / \mathrm{min}$ and an injection volume of $5.0 \mu \mathrm{l}$. A Finnigan LCQ DECA XP plus iontrap Mass Spectrometer (Thermo Corp., USA) was connected to the HPLC system via an electrospray ionization (ESI) interface. The ESI source was operated in positive and negative ionization mode with a capillary voltage of $4.0 \mathrm{kV}$. The cone and desolvation gas flows were $10 \mathrm{~L} / \mathrm{h}$.

\section{Taste evaluation of compounds 1-9}

Compounds 1-9 were dissolved in $80 \mathrm{ml}$ purified water (final concentration of the test solution, $9.6 \mathrm{mM}$ ). When the reference electrode and sensors were dipped into the 
test solution, a potentiometer difference between each individually coated sensor and the $\mathrm{Ag} / \mathrm{AgCl}$ reference electrode was recorded by the e-Tongue. Each compound was analyzed for $120 \mathrm{~s}$. The liquid sensors and the reference electrode were rinsed with purified water for $10 \mathrm{~s}$ inbetween analyses. Using well-conditioned sensors, each compound was analyzed seven or eight times by a rotation procedure (i.e., the first round of compound measurements was completed before the next round of compound measurements was initiated) [13].

\section{Minimum inhibitory concentration (MIC) determination $[17,18]$}

The antibacterial activities of compounds 1-9 were assessed by the microdilution method [19]. Compounds 1-9 were dissolved in DMSO (10\% of the final volume) and diluted with Mueller-Hinton broth to a concentration of $1 \mathrm{mg} / \mathrm{ml}$. Further dilutions (1:3) were performed with the addition of culture broth, reaching concentrations that ranged from 0.037 to $1 \mathrm{mg} / \mathrm{ml}$. An aliquot $(200 \mu \mathrm{l})$ of each diluted compound was added to a 96well plate. A growth control (containing MuellerHinton broth with DMSO, but without the compounds) and positive control drug (Kanamycin of $50 \mu \mathrm{g} / \mathrm{ml}$ ) was added to the 96-well plate. The compounds and growth control were inoculated with $5 \mu \mathrm{l}$ of the bacterial suspension and incubated at $36{ }^{\circ} \mathrm{C}$ for $24 \mathrm{~h}$. Bacterial growth was assessed by optical density. MIC was defined as the lowest concentration of each compound that inhibited bacterial growth.

\section{Data analyses}

Data were analyzed by principal component analysis (PCA) and partial least squares regression (PLS) $[9,11,13]$. PCA was used to estimate the largest and second largest relative contribution factors (i.e., PC1 and PC2) from the eTongue. The values were computed as a percentage by the Alpha MOS software. PC1 and PC2 explained the total variance of different samples and groups, respectively. Therefore, PCA assessed the bitterness of each isolated compound.

PLS was used to correlate the instrument data (sensor responses) with bitterness (bitterness intensity scores). On the PLS graph, the measurements corresponding to each compound (X-axis) were plotted against the instrumental data for each compound (Yaxis). A correlation was observed between the two data sets (solution and instrumental). The correlation coefficient $\left(R^{2}\right)$ of the PLS calibration curve should be equal or higher than 0.80 . When the mixture sample was determined, the instrumental measurements could be obtained by the e-Tongue; bitterness (quantified as limonin) was calculated by PLS.

\section{Results and Discussion}

\section{Phytochemistry of E. rutaecarpa}

The methanolic extract of E. rutaecarpa fruits was subjected to column chromatography over silica gel, RP-18, and Sephadex LH-20 in various solvent systems to afford five known indoloquinazoline alkaloids (1-5), two known quinazolinedione alkaloids (6 and 7 ), and two

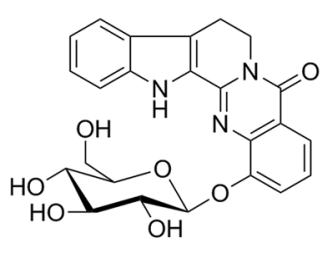

1<smiles>O=CN1c2ccccc2C(=O)N2CCc3c([nH]c4ccccc34)C21</smiles>

4
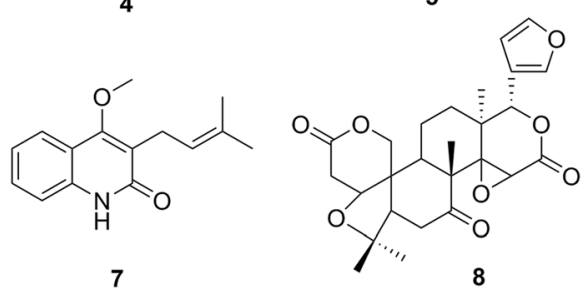

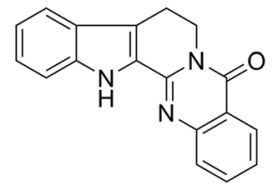

3<smiles>CCCC/C=C\CCCCCCCc1cc(=O)c2ccccc2n1C</smiles>

6

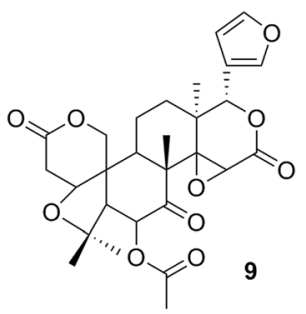

Fig. 1 Structures of compounds $\mathbf{1 - 9}$ 


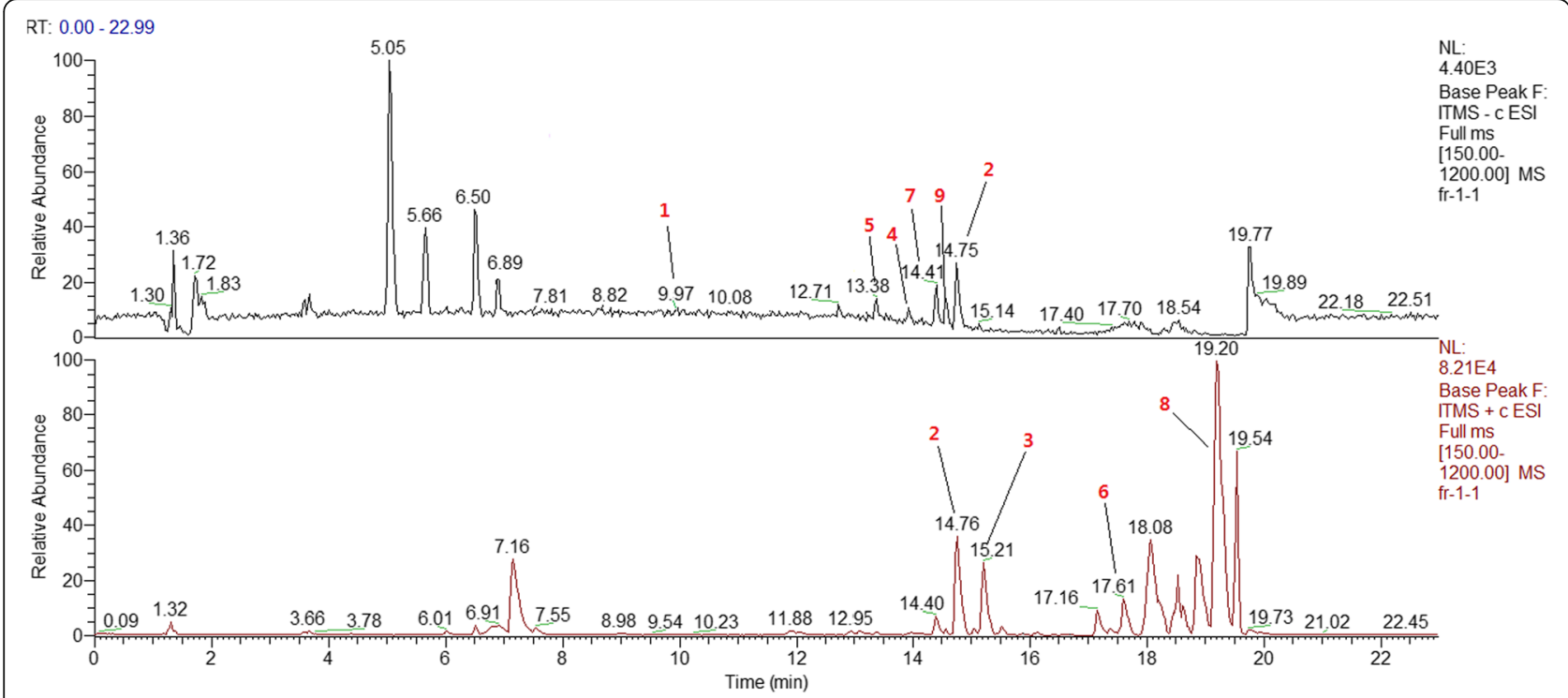

Fig. 2 HPLC-MS chromatogram of E. rutaecarpa extract

known limonoids (8 and 9). By comparing physical and spectroscopic data with reported data, the known compounds were identified as 1-O- $\beta$-D-glucopyranosylrutaecarpine (1) [20], evodiamine (2) [15], rutaecarpine (3) [21], 14-formyldihydro-rutaecarpine (4) [22], hydroxyevodiamine (5) [23], evocarpine (6) [24], 4-methoxy-3-(3methylbut-2-enyl)- $1 H$-quinolin-2-one (7) [25], limonin (8) [16], 6 $\beta$-acetoxy-5-epilimonin (9, Fig. 1, Additional file 1) $[26]$.

\section{Chromatographic and mass spectrometric results}

The chromatographic and mass spectrometric results of $E$. rutaecarpa are shown in Fig. 2. The contents of compounds 1-9 are shown in Table 1. Among the compounds, compound 8 was the most predominant (2.0864\%).

Table 1 Response values, bitterness relative to limonin, and contents of compounds $\mathbf{1 - 9}$

\begin{tabular}{llll}
\hline Compounds & $\begin{array}{l}\text { Response } \\
\text { Values }\end{array}$ & $\begin{array}{l}\text { Bitterness relative } \\
\text { to limonin }\end{array}$ & Content (\%) \\
\hline 1 & 3.691 & 0.377 & 0.0095 \\
2 & 3.483 & 0.356 & 0.4852 \\
3 & 4.499 & 0.459 & 0.3045 \\
4 & 4.689 & 0.478 & 0.0360 \\
5 & 4.154 & 0.424 & 0.1473 \\
6 & 0.712 & 0.076 & 0.2355 \\
7 & 2.081 & 0.214 & 0.2292 \\
8 & 9.717 & 0.986 & 2.0864 \\
9 & 8.901 & 0.904 & 0.1862 \\
\hline
\end{tabular}

\section{Electronic tongue evaluation}

The qualitative analysis result of PCA (Fig. 2) showed that the compounds in descending order of bitterness were $8>9>4>3>5>1>2>7>6$. The results revealed that limonoids are bitterer than indoloquinazoline alkaloids, which in turn are bitterer than quinolone alkaloids.

Limonin (8), a representative bitter natural compound, is a reference in PLS. Different concentrations of compound $8(1.4,2.8,5.3,9.4$ and $16.5 \mathrm{mM})$ were evaluated by the e-Tongue (Fig. 3). There was a significant positive linear relationship for 8 at concentrations $1.4-16.5 \mathrm{mM}$ $\left(\mathrm{Y}=1.018 \mathrm{X}-0.037, \mathrm{R}^{2}=0.999\right)$. The reference concentration was plotted on the $\mathrm{X}$-axis; the response value of the e-Tongue was plotted on the Y-axis. The R.S.D. values for all samples were $<5 \%$. These results suggest that the sensor variation was insignificant. The response values of the other eight compounds generated linear equations. The relative bitterness of compounds 1-9 are shown in Fig. 4.

\section{Bacteriostatic activity}

The result of antibacterial test (Table 2) suggested that all the nine compounds had antibacterial activities to some extent. Limonoids showed greater activities than alkaloids, and indoloquinazoline alkaloids had greater activities than quinolone alkaloids.

Based on the contents of all constituents and bacteriostatic activity intensity, limonin (8) was the bitterest contributive compound of all the ones from Evodia rutaecarpa. Therefore, potential interactions amongst limonoids, indoloquinazoline alkaloids and quinolone 


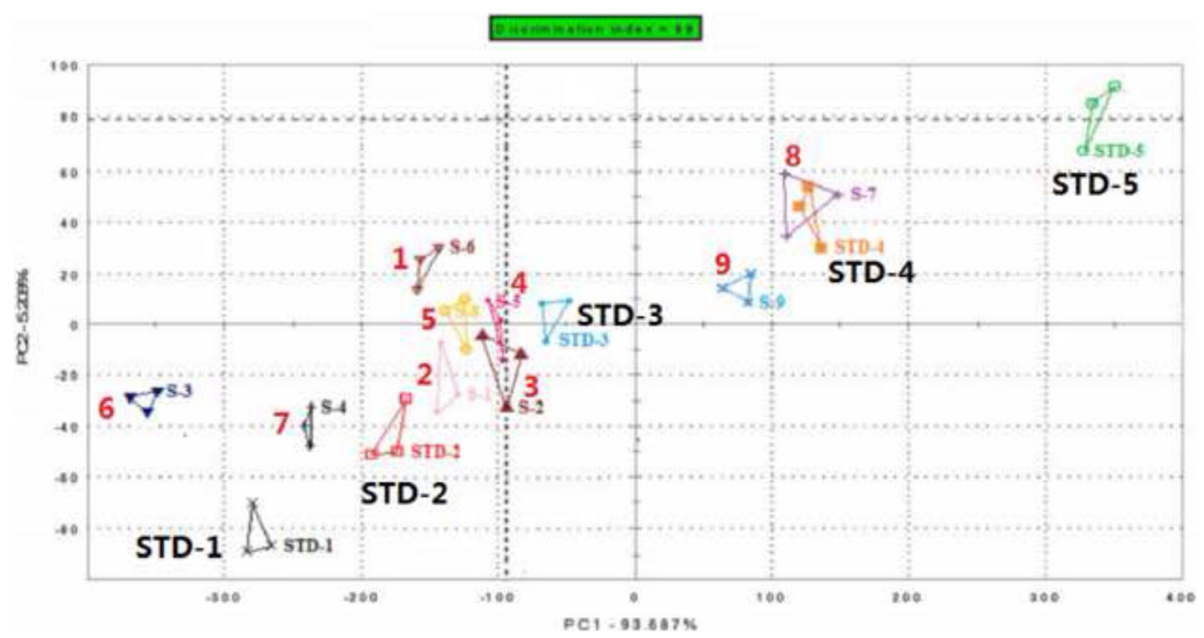

Fig. 3 Principal component analysis (PCA) by e-Tongue

alkaloids in bitterness contributions and antibacterial activity will be ahead the further direction of effort, such as synergistic, antagonistic, additive effect, etc.

In general, the modern electronic tongue technology with tremendous development potential play a more and more important role in the research of bitter herbs as well as wide applications in food industry and pharmaceutical analytics [6-12]. Yaroshenko [27] evaluated bitterness of eight various herbal medicine samples via three different approaches involving high-performance liquid chromatography coupled to UV detector, capillary electrophoresis coupled to UV detector and a potentiometric multisensor system - electronic tongue employing PLS regression and it was demonstrated that all three methods were able to be applied for quantitative assessment of bitterness in TCM with reasonable precision. Lin [28] constructed Robust Partial Least Squares (RPLS) model to evaluate the bitterness of a total of 35 TCM detoctions using an eTongue and that showed RPLS are capable of quantitatively characterizing bitterness. However, there is little study about application of electronic tongue to evaluate bitter constituents from TCM combined with pharmacological activity. Therefore, it is worth trying to explore and summarize for technological promotion of e-Tongue and pharmaceutical research from long-term perspective.

\section{Conclusions}

Our findings suggest that limonoids are bitterer than indoloquinazoline alkaloids, and indoloquinazoline

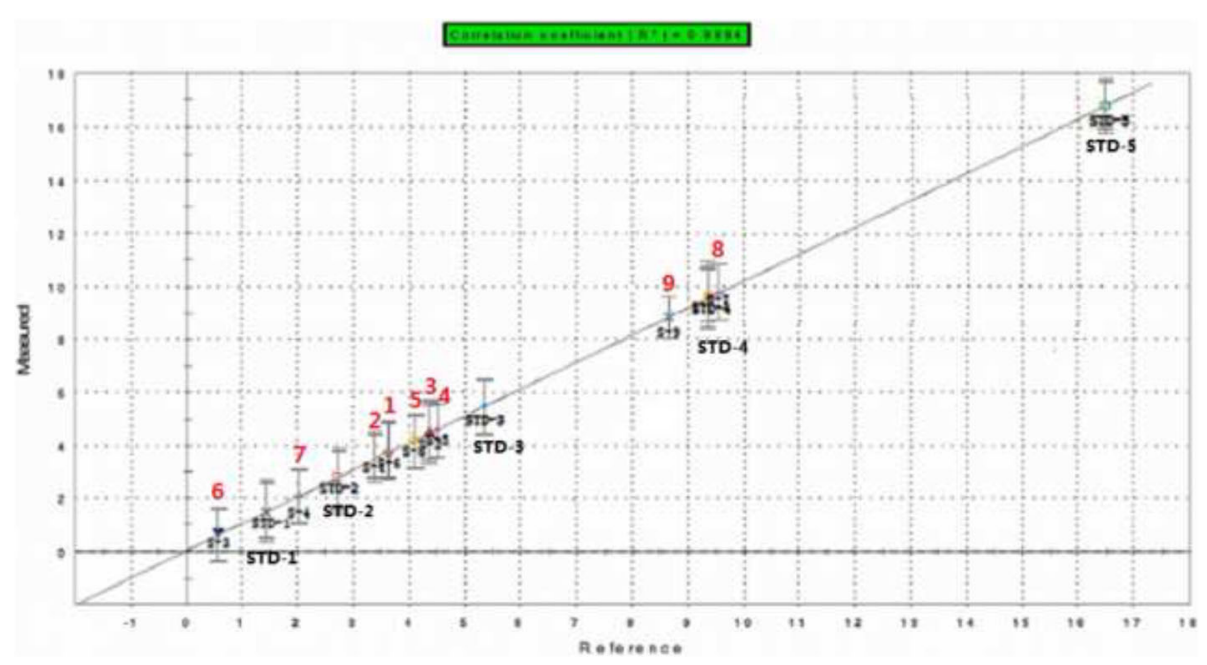

Fig. 4 Partial least squares (PLS) analysis by e-Tongue 
Table 2 Antibacterial activities of compounds $\mathbf{1 - 9}$ against E. coli and S. aureus

\begin{tabular}{|c|c|c|c|c|c|c|c|c|}
\hline \multirow[t]{2}{*}{ Compounds } & \multicolumn{4}{|l|}{ E. coli } & \multicolumn{4}{|l|}{ S. aureus } \\
\hline & $1 \mathrm{mg} / \mathrm{ml}$ & $0.33 \mathrm{mg} / \mathrm{ml}$ & $0.11 \mathrm{mg} / \mathrm{ml}$ & $0.037 \mathrm{mg} / \mathrm{ml}$ & $1 \mathrm{mg} / \mathrm{ml}$ & $0.33 \mathrm{mg} / \mathrm{ml}$ & $0.11 \mathrm{mg} / \mathrm{ml}$ & $0.037 \mathrm{mg} / \mathrm{ml}$ \\
\hline 1 & - & + & + & + & - & + & + & + \\
\hline 2 & - & - & + & + & - & + & + & + \\
\hline 3 & - & - & + & + & - & + & + & + \\
\hline 4 & - & + & + & + & + & + & + & + \\
\hline 5 & - & + & + & + & + & + & + & + \\
\hline 6 & - & + & + & + & + & + & + & + \\
\hline 7 & - & + & + & + & + & + & + & + \\
\hline 8 & - & - & - & + & - & - & - & + \\
\hline 9 & - & - & - & + & - & - & + & + \\
\hline
\end{tabular}

1. " + " represents bacteria can be observed in the experimental concentration; " - " represents bacteria can not be observed in the experimental concentration 2. Four compound concentration levels $(1 \mathrm{mg} / \mathrm{ml} 、 0.33 \mathrm{mg} / \mathrm{ml} 、 0.11 \mathrm{mg} / \mathrm{ml} 、 0.037 \mathrm{mg} / \mathrm{ml})$ represent four antibacterial activity intensities (0、1、2、3), respectively

alkaloids are bitterer than quinolone alkaloids. Considering their contents in E. rutaecarpa given by the results of LC-MS, limonoids are the main contributors to the bitter taste of the herb. In addition, alkaloids, especially indoloquinazoline alkaloids, are other important bitter constituents of E. rutaecarpa.

Similarly, the result of antibacterial test suggested limonoids had greater antibacterial properties than alkaloids, and indoloquinazoline alkaloids had greater antibacterial properties than quinolone alkaloids. Therefore, there is a positive correlation between bitter taste and antibacterial activities. Further studies are needed to confirm these findings.

\section{Additional file}

Additional file 1: The ${ }^{13} \mathrm{C}$ and ${ }^{1} \mathrm{H}$ NMR spectroscopic data of compounds 1-9. (DOC $58 \mathrm{~kb})$

\begin{abstract}
Abbreviations
COSY: Correlation spectroscopy; ELISA: Enzyme-linked immunosorbent assay; ESI: Electrospray ionization; ESI-MS: Electrospray ionization-mass spectrum; eTongue: Electronic Tongue; HMBC: Heteronuclear multiple-bond correlation; HPLC: High performance liquid chromatography; HR-ESI-MS: High resolution ESI-MS; HSQC: Heteronuclear single quantum coherence; IR: Infrared spectrometer; LB: Lysogeny broth; LC/MSD: Liquid chromatography/mass spectrometer detector; LC-MS: Liquid chromatography-mass spectrum; MIC: Minimum inhibitory concentration; NMR: Nuclear magnetic resonance; NOESY: Nuclear overhauser enhancement spectroscopy; PBS: Phosphatebuffered solution; PCA: Principal component analysis; PLS: Partial least squares; Q-TOF: Quadrupole-time-of-flight; R.S.D.: Relative standard deviation; TCM: Traditional Chinese Medicine; TMS: Tetramethylsilane
\end{abstract}

\section{Acknowledgments}

This research was support by Second Military Medical University and we would like to thank those colleagues who helped us to carry out this study.

\section{Funding}

The work was supported by the funds from Shanghai Municipal Commission of Health and Family Planning (ZY3-CCCX-3-5001) and Science and Technology Commission of Shanghai Municipality (15DZ2292000).

Availability of data and materials

The datasets supporting the conclusions of this article are included within the article and the additional file.

\section{Authors' contributions}

$X G L, B L$ and SL provided the innovative ideas and designed this study; XGL and BL performed the research and wrote the paper; FW and TZL participated in data collection and statistical analysis; YJW was involved in the experiments and QM coordinated the study; SL reviewed the final draft of the manuscript. All authors read and approved the final manuscript.

\section{Competing interests}

The authors declare that they have no competing interests.

Consent for publication

Not applicable.

Ethics approval and consent to participate

Not applicable.

\section{Publisher's Note}

Springer Nature remains neutral with regard to jurisdictional claims in published maps and institutional affiliations.

\section{Author details}

'Engineering Research Center of Modern Preparation Technology of TCM, Ministry of Education, Shanghai University of Traditional Chinese Medicine, 1200 Cailun Road, Shanghai 201203, People's Republic of China. ${ }^{2}$ Amway (China) R \& D Center Co., Ltd., Shanghai 201203, People's Republic of China. ${ }^{3}$ Amway (China) Botanical R \& D Center Co., Ltd., Wuxi 214115, People's Republic of China.

Received: 8 December 2016 Accepted: 23 March 2017

Published online: 29 March 2017

\section{References}

1. Pharmacopoeia NCoC: Pharmacopoeia of the People's Republic of China. Beijing: Chemical Industry Press; 2010

2. Feng X, Yan D, Zhao KJ, Luo JY, Ren YS, Kong WJ, Han YM, Xiao XH. Applications of microcalorimetry in the antibacterial activity evaluation of various Rhizoma coptidis. Pharm Biol. 2011;49(4):348-53. 
3. Li WY, Chan SW, Guo DJ, Chung MK, Leung TY, Yu PH. Water extract of Rheum officinale Baill. induces apoptosis in human lung adenocarcinoma A549 and human breast cancer MCF-7 cell lines. J Ethnopharmacol. 2009;124(2):251-6.

4. Wang $X N$, Xu LN, Peng JY, Liu KX, Zhang LH, Zhang YK. In vivo inhibition of S180 tumors by the synergistic effect of the Chinese medicinal herbs Coptis chinensis and Evodia rutaecarpa. Planta Med. 2009;75(11):1215-20.

5. Xia X, Yan J, Shen Y, Tang K, Yin J, Zhang Y, Yang D, Liang H, Ye J, Weng J. Berberine improves glucose metabolism in diabetic rats by inhibition of hepatic gluconeogenesis. PLoS One. 2011;6(2):e16556.

6. Dominguez RB, Moreno-Baron L, Munoz R, Gutierrez JM. Voltammetric electronic tongue and support vector machines for identification of selected features in Mexican coffee. Sensors (Basel). 2014;14(9):17770-85.

7. Dias LG, Sequeira C, Veloso AC, Sousa ME, Peres AM. Evaluation of healthy and sensory indexes of sweetened beverages using an electronic tongue. Anal Chim Acta. 2014;848:32-42.

8. Bagnasco L, Cosulich ME, Speranza G, Medini L, Oliveri P, Lanteri S. Application of a voltammetric electronic tongue and near infrared spectroscopy for a rapid umami taste assessment. Food Chem. 2014;157:421-8.

9. Lorenz JK, Reo JP, Hendl O, Worthington JH, Petrossian VD. Evaluation of a taste sensor instrument (electronic tongue) for use in formulation development. Int J Pharm. 2009:367(1-2):65-72.

10. Legin A, Rudnitskaya A, Clapham D, Seleznev B, Lord K, Vlasov Y. Electronic tongue for pharmaceutical analytics: quantification of tastes and masking effects. Anal Bioanal Chem. 2004;380(1):36-45.

11. Kataoka M, Tokuyama E, Miyanaga Y, Uchida T. The taste sensory evaluation of medicinal plants and Chinese medicines. Int J Pharm. 2008;351(1-2):36-44.

12. Harada T, Uchida T, Yoshida M, Kobayashi Y, Narazaki R, Ohwaki T. A new method for evaluating the bitterness of medicines in development using a taste sensor and a disintegration testing apparatus. Chem Pharm Bull (Tokyo). 2010;58(8):1009-14.

13. Wang $Y$, Feng $Y, W u Y$, Liang $S, X u$ D. Sensory evaluation of the taste of berberine hydrochloride using an Electronic Tongue. Fitoterapia. 2013;86:137-43.

14. Liao JF, Chiou WF, Shen YC, Wang GJ, Chen CF. Anti-inflammatory and antiinfectious effects of Evodia rutaecarpa (Wuzhuyu) and its major bioactive components. Chin Med. 2011;6(1):6-13.

15. Xu M-L, Moon D-C, Lee C-S, Woo M-H, Lee E, Jahng Y, Chang H-W, Lee S-H, Son J-K. Cytotoxicity and DNA topoisomerase inhibitory activity of constituents isolated from the fruits of Evodia officinalis. Arch Pharm Res. 2006;29(7):541-7.

16. Sugimoto T, Miyase T, Kuroyanagi M, Ueno A. Limonoids and quinolone alkaloids from Evodia rutaecarpa Bentham. Chem Pharm Bull (Tokyo). 1988:36(11):4453-61.

17. Sadiq A, Ahmad S, Ali R, Ahmad F, Ahmad S, Zeb A, Ayaz M, Ullah F, Siddique AN. Antibacterial and antifungal potentials of the solvents extracts from Eryngium caeruleum, Notholirion thomsonianum and Allium consanguineum. BMC Complement Altern Med. 2016;16(1):478.

18. Yang Y, Ye XL, Li XG. Antimicrobial effect of four alkaloids from Coptidis Rhizome. Lishizhen Med Mater Med Res. 2007;18(12):3013-4.

19. Valgas C, Souza SM, Smânia EFA, Smânia Jr A. Screening methods to determine antibacterial activity of natural products. Braz J Microbiol. 2007;38:369-80.

20. Xia X, Luo JG, Liu RH, Yang MH, Kong LY. New alkaloids from the leaves of Evodia rutaecarpa. Nat Prod Res. 2016:1-6.

21. D'Yakonov AL, Telezhenetskaya MV. Quinazoline alkaloids in nature. Chem Nat Compounds. 1997;33(3):221-67.

22. Guoying Zuo HH, Wang B, Hong X, Hao X. A new indoloqiunazoline alkaloid from the fruit of Evodia rutaecarpa. Acta Bot Yunnanica. 2003;25(1):103-6.

23. Yuanqing Tang XFHL. Studies on the chemical constituents of evodia rutaecarpa [Juss] Benth. J Chin Pharm Sci. 1997;6(2):65-9.

24. Tang $Y-Q$, Feng $X-Z$, Huang L. Quinolone alkaloids from Evodia rutaecarpa. Phytochemistry. 1996;43(3):719-22.

25. Jones $K$, Roset X, Rossiter S, Whiffield P. Demethylation of 2,4-dimethoxyquinolines: the synthesis of atanine. Org Biomol Chem. 2003;1(24):4380-3.

26. Sugimoto T, Ueno A, Kadota S, Cui C, Kikuchi T. New $5 \beta$-H limonoids from evodia rutaecarpa bentham. Chem Pharm Bull (Tokyo). 1988;36(3):1237-40.

27. Yaroshenko I, Kirsanov D, Kartsova L, Sidorova A, Sun Q, Wan H, He Y, Wang P, Legin A. Exploring bitterness of traditional Chinese medicine samples by potentiometric electronic tongue and by capillary electrophoresis and liquid chromatography coupled to UV detection. Talanta. 2016;152:105-11.

28. Lin Z, Zhang Q, Liu R, Gao X, Zhang L, Kang B, Shi J, Wu Z, Gui X, Li X. Evaluation of the Bitterness of Traditional Chinese Medicines using an ETongue Coupled with a Robust Partial Least Squares Regression Method. Sensors. 2016;16(2):151.

\section{Submit your next manuscript to BioMed Central and we will help you at every step:}

- We accept pre-submission inquiries

- Our selector tool helps you to find the most relevant journal

- We provide round the clock customer support

- Convenient online submission

- Thorough peer review

- Inclusion in PubMed and all major indexing services

- Maximum visibility for your research

Submit your manuscript at www.biomedcentral.com/submit 